\title{
Modular Installation for Electromechanical Processes
}

\author{
Adrian T. Plesca* \\ Department of Power Engineering, Gheorghe Asachi Technical University of lasi, Romania; \\ matrix_total2000@yahoo.com
}

\begin{abstract}
This paper presents a modular multifunctional installation for electromechanical workshops and manufacture departments or technical assistance to provide the accomplishment of the specific processing operating. The main component parts of this installation are described such as: devices type transformer-stabilizer, multiple converters of electrical energy into magnetic, thermal and mechanical energy. There are described also, some new types of electrothermal sources for supplementary heating in the case of electromechanical processing. These thermal sources can be made with electromagnet component parts, traditional asynchronous rotating motors or with arch stator (or double arch stator) linear motors. Finally, the advantages of this new installation have been outlined.
\end{abstract}

Keywords: Modular Installation, Energy Converters, Thermal Sources.

\section{Introduction}

During electromechanical processes result an important quantity of heat because of metal deformation and tool piece friction and it adds the specific heat in the contact resistance between different type of electric pieces. This local heat decreases the metal resistance and so, the electromechanical manufacturing process is easily to be done. The obtained heat in the working area depends on the operating speed, tool pressure force and the current density through different electric components. A few previous works had investigated the electrothermal analysis due to electromechanical processes. Instead, there are many papers related to thermal analysis of power electronic devices and circuits. A method that offers a powerful alternative to conventional numerical thermal simulation techniques, and is constructed to be compatible with explicitly coupled electrothermal device and circuit simulation on CAD timescales, is presented in [1]. In contrast to semianalyti$\mathrm{cal}$, frequency space, the method is based on explicit, fully analytical, double Fourier series expressions for thermal subsystem solutions in Laplace transform s-space (complex frequency space). In [2], a portable subcircuit for electronic components as bipolar transistors, is developed that incorporates self heating as well as thermal coupling effects. Thermal parameters are modelled using thermal-electrical analogy. Finite difference approach is used to model the thermal grid network. A new methodology is presented in [3] for constructing thermal equivalent circuits for electrothermal simulation with a multitude of temperature dependent heat sources. The method is applied to a high frequency dc/dc-converter and a dc/ac-converter module both for automotive applications. The approach presented in [4] yields modular medium-order models for extensive and complicated geometries, such as a power-electronic chip. Moreover, representing the thermal behaviour with an equivalent circuit enables coupled simulations of electrothermal behaviour, which are important in power electronics. The development and implementation of an analytical 3-D thermal model for fast and accurate thermal simulation of power device modules in electrothermal converter simulation is described in [5] and a transient electro-thermal analysis considering self-heating is shown in [6]. A model of a semiconductor converter-fed dual-stator windings induction machine of the adjustable-speed propulsion drive is presented in [7]. It is built up by decomposing

*Corresponding author:

Adrian T. Plesca (matrix_total2000@yahoo.com) 
the system into sub-circuits with lumped parameters which are coupled together by means of dependent voltage/ current sources. In [8], a new method of SPICE macromodeling of magnetic components, that considers both the magnetic saturation and hysteresis processes, as well as the self-heating electrothermal effects, is presented. An electrothermal analysis of a plasma source using a 2D approach is shown in [9]. The 3D temperature distribution in a dry type transformer, including heat generation by Joule losses and eddy currents, as well as natural cooling on the outside of the metal casing, is presented in [10]. Calculation models for electromagnetic field and temperature analysis for both stator and rotor in the case of an induction motor with compound cage rotor, are established in [11]. Heating of machines is a key characteristic of their behaviour. Heat sources come mainly from energy losses during electromechanical conversions (electrical in copper, magnetic in iron, aerolic and mechanical in bearings). Temperatures changes can be described thanks to thermal conduction, convection and radiation equations. Thus, it is necessary to build a thermal model adapted to the simulation and sizing of electrical generators, [12]. Two electro-thermal numerical models to be used for the modelling and optimization of high currents DC busbar contacts, are described in [13]. The heat sources can be used to obtain new ferroelectrets, [14]. Induction heating technology is nowadays widely present in domestic appliances because of its cleanness, high efficiency, and faster heating times. All of these advantages are due to its heating process, where the pot is directly heated by a varying magnetic field, [15]. Electromechanical coupling has been widely used to actuate and control micromechanical structures and to produce bulk nanostructured materials from micron and submicron particles. The understanding of the mechanical deformation of a mechanical structure carrying an electric current requires the analyses of electric and current-induced thermomechanical fields, [16, 17]. A coupled electromechanical approach can be used to analyse the energy transfer and consumption in adaptive structures, $[18,19]$. In contrast to classical object-oriented electromechanical modelling, i.e. describing plates, suspensions, drives, etc., it proposes in [20-24], to combine models of basic physical effects to object models which in turn can be used for system modelling.

The modular multifunctional installation has been designed with the aim to be included into the flexible processing systems where the electromechanical processing is necessary, but also, this installation can be used at workshops and autonomous electromechanical departments.
The component parts of this installation have a modular design in order to achieve different types of installations with any necessary modules and all the modules may carry out different type of functions. Hence, the individual and group assembly has an increased utilization coefficient and in the same time the expenses during all intermediary steps of the electromechanical process, are decreased.

The nowadays installations used in different type of electromechanic workshops have the following drawbacks: huge volumes, big costs and there is the necessity of the high qualification staff, great consumption of the active materials and small utilization coefficient, reduced workable, very difficult to be transported, small degree of adaptation, low flexibility. In order to avoid the above drawbacks, a new type of modular multifunctional installation has been designed and is presented in this paper.

\section{The Description of the Modular Multifunctional Installation}

The electromechanical workshops and specialized departments in manufacturing processes are very important component parts from:

- automation industry: specialized departments to accomplish electromechanical processes;

- enterprises and production departments whose purpose of activity is the assembling, maintenance and running operations;

- as component parts for automotive maintenance and reparations, inside automatic plant transport and in the transportation field, in general.

The electromechanical workshops and departments perform a specific group of operations which belong of some combined applications of electrotechnics and mechanics field. The electric department of the technological equipment includes adjusted current and voltage sources of DC or AC current, with stabilized values for an important range of values, and also some special thermal sources are included.

The main objective of this study was to provide a modular multifunctional installation with different and many applications in the huge field of electromechanics. The goal was to offer the ability of adaptation, maximum flexibility, efficiency and quality of operations using this new type of modular installation for different electromechanical processes. Further on, some achievements are presented. 
It has been designed a modular electromagnetic device which works as transformer or stabilizer, built for a certain rated power, voltage and current ranges. Also, it works as a multiple converter of electromagnetically, thermal, mechanical energy and as electrothermal source.

The new features of this modular multifunctional installation are the followings:

- multifunctional electromagnetic devices with double function: voltage transformer - stabilizer and current transformer - stabilizer, Figure 1 and Figure 2;

- multiple converters of electrical energy into magnetically, thermal and mechanical (vibrations, rotates and translations) energy, Figure 3;

- electrothermal sources for electromechanical processes for pieces with cylindrical or plane shapes, Figures 4-7.

The multifunctional electromagnetic device type voltage transformer - stabilizer, has the magnetic core $\mathrm{m}$, built from two pieces: the fixed armature $A_{f}$ made from sheet plates type $\mathrm{E}$, and the moving armature $\mathrm{A}_{\mathrm{d}}$, using sheet plates type I (sliding with the gap $\delta$, in order to achieve the stabilization function). Both armature are stiffed using mechanical accessories. The primary P, of the transformer, Figure 1a, and the secondary S, have fixed turns terminals. To extend the rated voltage, a mechanical switch can be mounted with the aim to add or shortcircuit a certain numbers of windings/turns. Therefore, all necessary rated voltages will be achieved. The same situation is presented in the case of stabilization function, Figure 1b, where can be observed the primary $\mathrm{P}$, secondary $\mathrm{S}$, compensation winding $\mathrm{k}$ and the capacitive winding $\mathrm{c}$. In order to extend the possibilities to obtain different secondary voltages, a double switch $\mathrm{k}_{1}-\mathrm{k}_{2}$, Figure $1 c$, is required. For a higher rated power, a series of transformers-stabilizers, each module with a certain rated power, can be constructed.

The device type current transformer - stabilizer has the magnetic circuit type UI, and it observes the windings $P$ and S, Figure 2a, which can be made from two or more windings with a certain number of turns. The magnetic shunt $\mathrm{m}_{\mathrm{p}}$, has the pre-magnetization function and it uses DC current source. The goal is to obtain an adjusted current within a certain range.

The magnetic shunt, Figure $2 \mathrm{~b}$, has a special construction: the magnetic circuit $m_{p}$, Figure $2 b$, is made from a circular strap with rectangular cross-section, Figure 2c. Inside its window $F$, is placed the control coil $b_{c}$, for DC orthogonal pre-magnetization which generates the
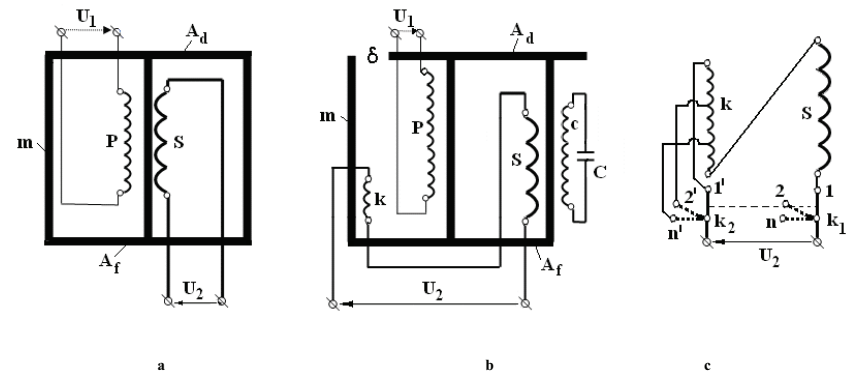

Figure 1. The electromagnetic device type voltage transformer-stabilizer.

magnetic field $\mathrm{H}_{c}$, perpendicularly on the working magnetic field direction $\mathrm{H}_{\mathrm{p}}$, in any point of its volume.

With non pre-magnetized shunt and placed at higher position it gets the transformer function, and with premagnetized shunt the adjusted current stabilizer function is achieved.

The constructive solutions for multiple converters of electrical energy into other energy forms, are shown in Figure 3. It is presented the basics of a triple converter of electrical energy into magnetically, thermal and mechanical energy. The secondary S, vibrates when the primary $\mathrm{P}$, is supplied (the vibrations are because of radial and axial electromagnetic forces). Using special constructions for the secondary $\mathrm{S}$, there is the possibility to obtain many different technical solutions. The core $\mathrm{m}$, has two pieces: fixed armature $A_{f}$ and moving armature $A_{d}$.

\section{Constructive Solutions for Supplementary Heating}

The mechanical process becomes an electromechanical one if it uses the electrical energy in a favourable way at tool - piece interaction area. The developed heat because of contact resistance power loss can be used as a supplementary energy with the aim to improve the initial mechanical process. In some cases, there is the necessity for a supplementary heating and can be obtained using electromagnetically devices. The goal is to produce a triple conversion of electrical energy into thermal, magnetic and mechanical one. Further on, some new constructive solutions to obtain supplementary heating are presented. In the Figure 4 is shown a single-phase electromagnetic device with magnetic core made from sheets. It includes a fixed armature $A_{f}$ and a moving armature $A_{d}$. Between the moving armature and the piece $\mathrm{P}$ to be manufactured, there is a minimum gap $\delta$ to allow the piece rotation. 


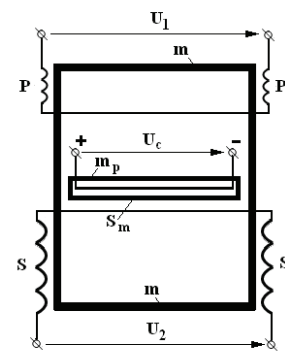

a)

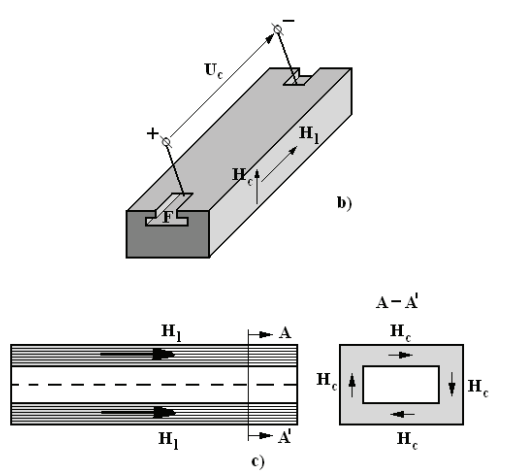

Figure 2. The electromagnetic device type current transformer-stabilizer.

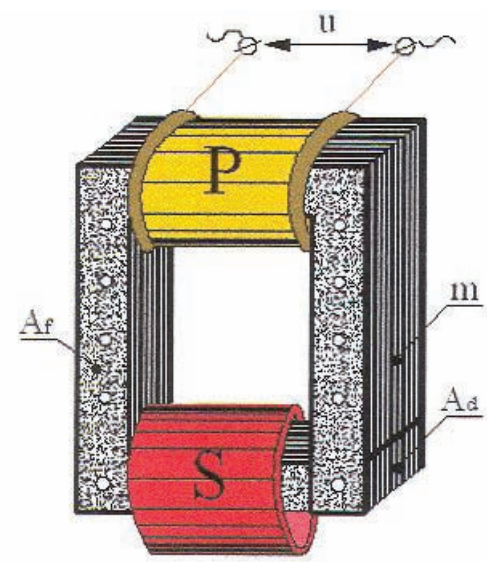

Figure 3. Multiple converter.

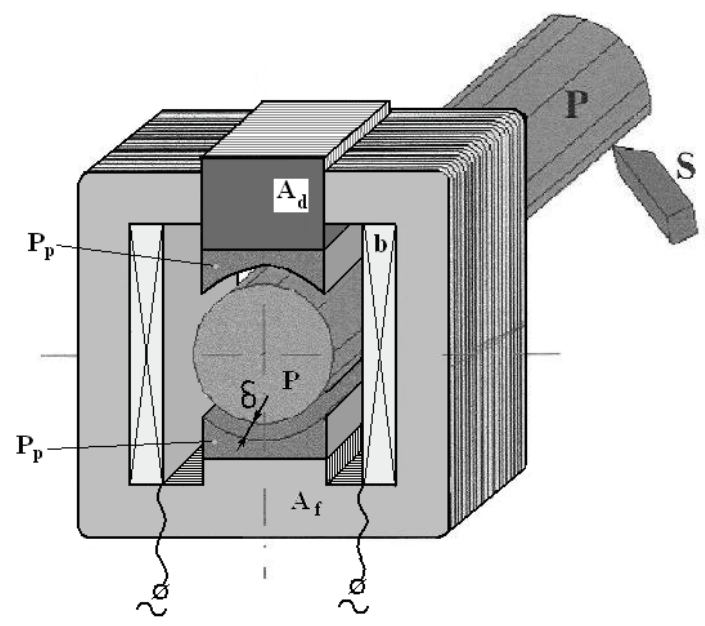

Figure 4. Single-phase electromagnetic device.

The electromagnetic device has also two bias pieces $\mathrm{P}_{\mathrm{p}}$, with different shapes depending on the processed piece $\mathrm{P}$. The coil $b$, crossed by the variable alternative current, provides a magnetic field with the induction $B$, and it obtains an electromotive force into piece. Hence, it obtains the heating because of the eddy currents. Therefore, it may uses a constant current source with the aim to achieve the necessary temperature for the piece $P$. The magnetic field through the volume of the piece, will lead to a quality improvement of the processed surface. At a certain frequency of the electric current, it obtains a certain penetration depth of the magnetic field and a specific heating because of the eddy currents.

In the Figure 5 it presents a solution using the stator $\mathrm{M}$, of the three-phase asynchronous or synchronous motors, where as rotor there is the piece $\mathrm{P}$, processed by the tool $\mathrm{S}$. The tree-phase stator can be supplied as a single-phase motor without capacitor, in order to avoid the piece rotating. The supplementary heat depends on the power supply system, eddy currents from the volume of the piece to be manufactured and the magnetic field.

If the stator with the proper windings, is cross sectioned, it gets, Figure 6, two arch stators, $M_{a 1}$ and $M_{a 2}$. The common rotor for both arch stators, is the piece P processed by the tool $\mathrm{S}$. These two identical stators can be supplied in order to get a null torque on the piece $\mathrm{P}$, and the gap $\delta$, can be adjusted with a special mechanical device. Using an adjusted current source it obtains the necessary heat for the piece $\mathrm{P}$. Therefore, the piece $\mathrm{P}$ will be processed in a better thermo-mechanical conditions.

In Figure 7, the magnetic field and supplementary heat for the piece $\mathrm{P}$, is obtained with a single-phase or threephase linear motor device. The heat amount depends on the RMS value of an adjusted current source. The power supply can be done in the order to obtain a pulse magnetic field with the aim to avoid the piece suppression and the moving because of device electromagnetic force.

\section{Experimental Tests}

The electrothermal sources for supplementary heating at electromechanical processes, in the case of iron, steel or cast iron components can be in the same time the magnetic field source which increases the operating qualities of the manufactured pieces. If it knows the magnetic characteristics (hysteresis cycle) of the processed material, the frequency and the magnetomotive force it can be evaluated the penetration depth and the power losses used at the heating and magnetic field treatment. Because of the penetration depth of the magnetic field inside the volume of the manufactured piece, it obtains the power losses because of the eddy currents and hysteresis cycle of the material. 


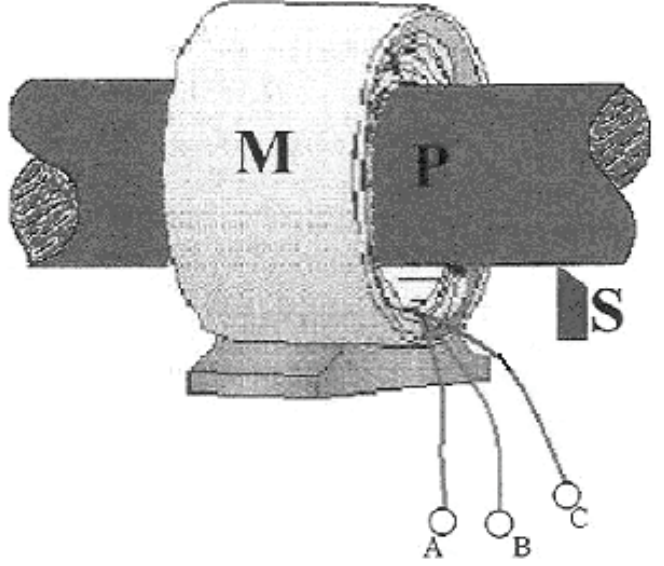

Figure 5. Device with stator of asynchronous motor.

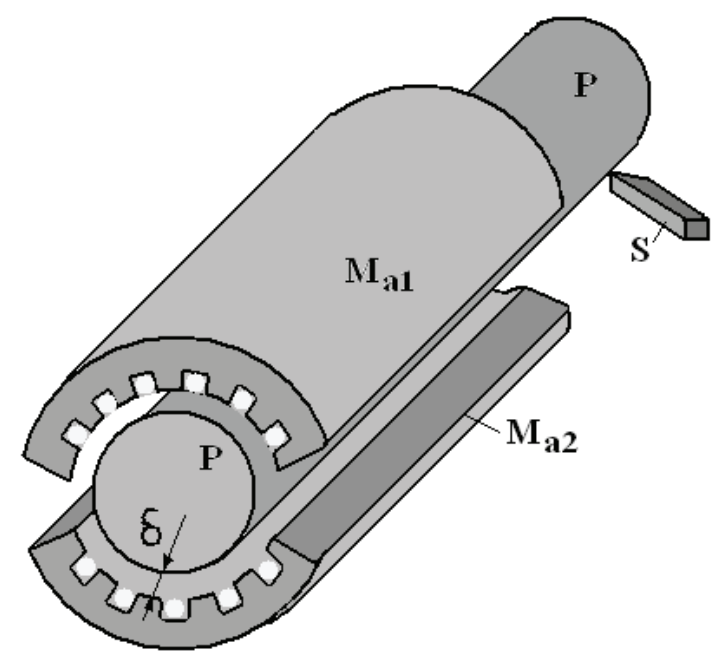

Figure 6. Device with two arch stators.

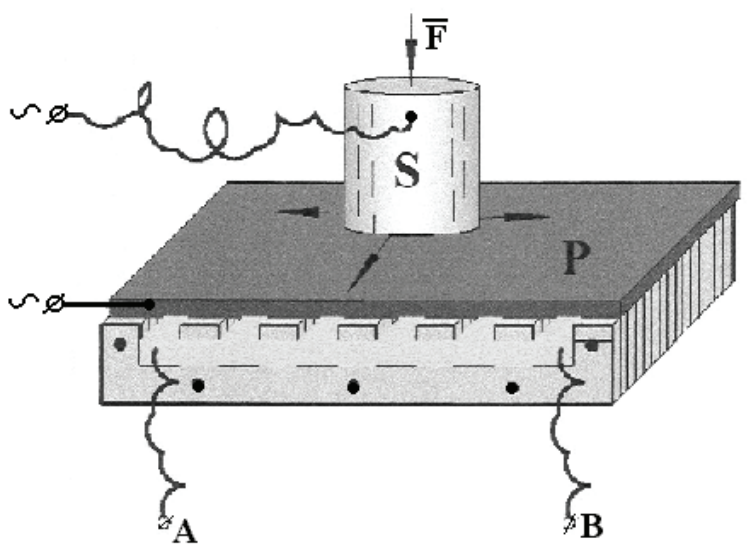

Figure 7. Device with linear motor.
This can be evaluated, and therefore knowing the thermal sources there is the possibility to estimate the heating within steady-state and transient conditions.

Further on, it presents the experimental test results obtained with an electromagnet $\mathrm{E}_{\mathrm{M}}$, Figure 8, where the moving armature is the processed piece $\mathrm{P}$, and using a three-phase stator of the asynchronous motor M, Figure 10 , where the rotor is the piece $P$ to be manufactured.

The experimental tests have outlined the following aspects:

- the heating (curves 1,2 and 3) and the cooling (curve 4) of the processed piece, are time exponential functions, Figure 8 and the steady-state temperature is reached after $4 \mathrm{~T}=40 \mathrm{~min}$. $(\mathrm{T}=10 \mathrm{~min}$, the time thermal constant). The heating curves have been obtained when the electric parameters for the electromagnet were: $\mathrm{U}=60 \mathrm{~V}$,

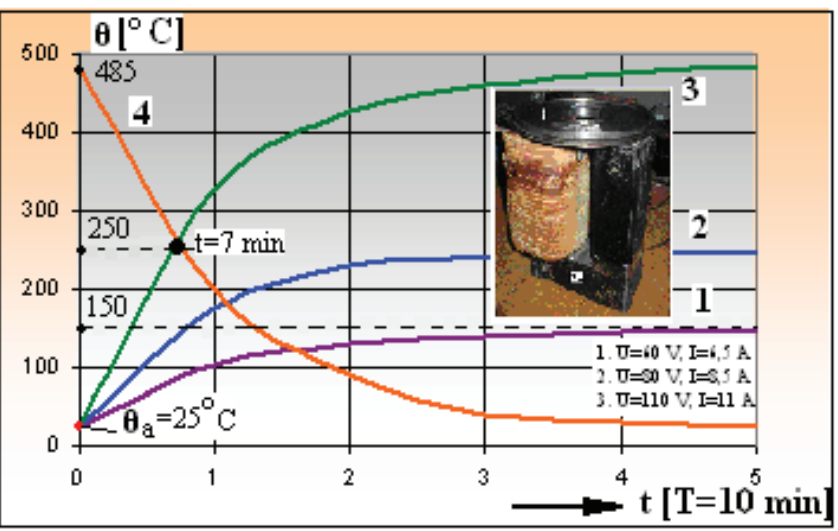

Figure 8. Thermal characteristics.

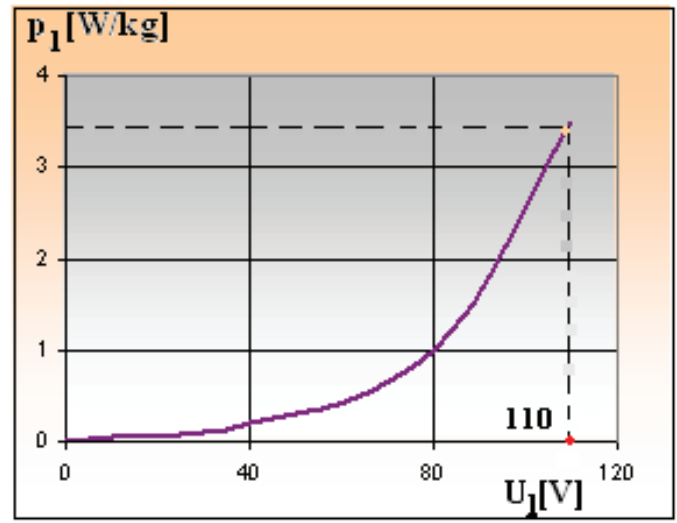

Figure 9. The characteristics of power loss and temperature vs. voltage. 


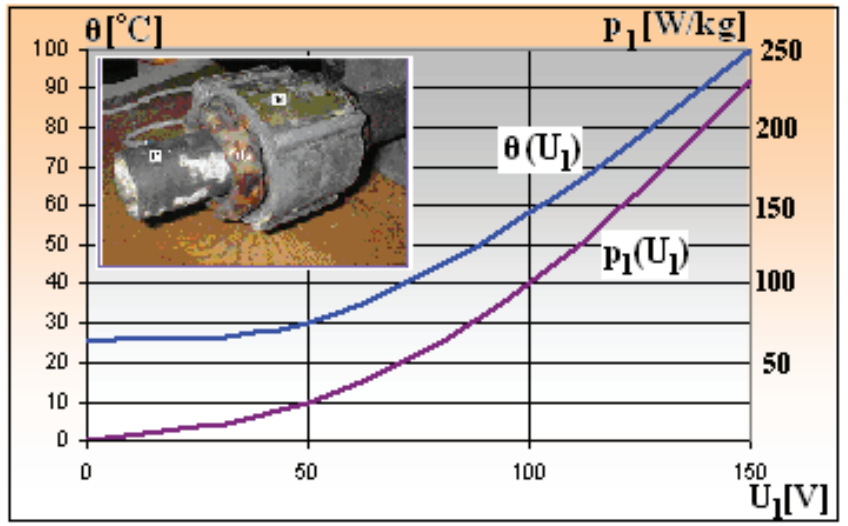

Figure 10. The characteristics of temperature and power loss vs. voltage in the case of rotate magnetic field.

$\mathrm{I}=6.5 \mathrm{~A}$ (curve \#1), $\mathrm{U}=80 \mathrm{~V}, \mathrm{I}=8.5 \mathrm{~A}$ (curve \#2) and $\mathrm{U}=110 \mathrm{~V}, \mathrm{I}=11 \mathrm{~A}$ (curve \#3);

- the specific iron power loss $p_{1}$ into electromagnet fixed armature $E_{M}$, the curve $p_{1}(U)$, Figure $9 a$, doesn't exceed $3.5 \mathrm{~W} / \mathrm{kg}$, which is within normal values (rated voltage of the electromagnet is $110 \mathrm{~V}$ );

- the power loss $\mathrm{p}_{1}$ in the piece $\mathrm{P}$, is higher than in the electromagnet, Figure 9b, which explains the higher temperatures, so there is the possibility to obtain a supplementary heating;

- the curve $\theta(U)$, Figure $9 c$, shows that the heat variation of the piece $\mathrm{P}$, can be adjusted depending on the voltage supply of the electromagnet $\mathrm{E}_{\mathrm{M}}$;

- the piece $\mathrm{P}$, vibrates and hence, during processing time the supplementary heat can be stopped;

- the heating within rotate magnetic field is similarly, from both temperature and power loss variation point of view, Figure 10;

- the solution with rotate magnetic field offers the following advantages: symmetrical load of the three-phase supply; the piece doesn't vibrates if it is well centred; the heating enforcement can be obtained; the increased heat can be changed depending on the direction of the rotate magnetic field; there is a more uniform heating on the surface of the manufactured piece.

\section{Conclusions}

The researches which have been made in this field, outline the following conclusions:
- the workshops and electromechanical departments can be equipped with a modular multifunctional installation to assure an efficient operating process within integrated automation and electrical engineering industry, with the aim to make more competitive the industrial companies through improvement the quality of their products;

- the new features of the modular multifunctional installation are: modular polyfunctional electromagnetic device; special device for supplementary heating at electromechanical processes; solutions with adjustable current sources to control the heating process;

- in the same time with the heating of the working place, the presence of the magnetic field may improve the quality of the processed surface of the piece to be manufactured;

- the heating source can be done in single-phase or threephase version, with the frequency of $50 \mathrm{~Hz}$ or more, and it works with pulse or rotate magnetic field.

\section{Acknowledgements}

This work was supported by CNCSIS - UEFISCDI, project number 610 PNII - CAPACITATI, 2013.

\section{References}

1. Batty W, Christoffersen C E et al. (2001). Electrothermal CAD of power devices and circuits with fully physical time-dependent compact thermal modeling of complex nonlinear 3-d systems, IEEE Transactions on Components and Packaging Technologies, vol 24(4), 566-590.

2. Shelar T S, and Visweswaran G S (2004). Inclusion of thermal effects in the simulation of bipolar circuits using circuit level behavioral modelling, 17th International Conference on VLSI Design, 821-826.

3. Gerstenmaier Y C, and Wachutka G K M (2004). Efficient calculation of transient temperature fields responding to fast changing heat sources over long duration in power electronic systems, IEEE Transactions on Components and Packaging Technologies, vol 27(1), 104-111.

4. Karagol S, and Bikdash M (2010). Generation of equivalentcircuit models from simulation data of a thermal system, IEEE Transactions on Power Electronics, vol 25(4), 820-828.

5. Swan I, Bryant A et al. (2012). A fast loss and temperature simulation method for power converters, Part II: 3-D thermal model of power module, IEEE Transactions on Power Electronics, vol 27(1), 258-268. 
6. Holzer S, Hollauer C et al. (2006). Three-dimensional transient interconnect analysis with regard to mechanical stress, 13th International Symposium on the Physical and Failure Analysis of Integrated Circuits, 154-157.

7. Pronin M, Shonin O et al. (2008). A model of semiconductor converter-fed asynchronous machines taking into account energy losses and thermal processes, 13th Power Electronics and Motion Control Conference, 837-844.

8. Maxim A, Andreu D et al. (1998). A novel behavioral method of SPICE macromodeling of magnetic components including the temperature and frequency dependencies, Thirteenth Annual Applied Power Electronics Conference and Exposition, vol 1, 393-399.

9. Winfrey L, Gilligan J et al. (2011). An investigation into radial gradients in an eletrothermal plasma source using a semi 2-D approach, IEEE International Conference on Plasma Science, 1.

10. Ortiz C, Skorek A et al. (2002). Parallel analysis of electrothermal phenomena in a dry type distribution, International Conference on Parallel Computing in Electrical Engineering, 381-385

11. Weili L, Junci C et al. (2010). Electrothermal Analysis of Induction Motor With Compound Cage Rotor Used for PHEV, IEEE Transactions on Industrial Electronics, vol $57(2), 660-668$

12. Maloberti O, Ospina A et al. (2012). Thermal modelling of a claw-pole car alternator: Steady-state computation and identification of free convection coefficients, XXth International Conference on Electrical Machines, 1888-1892.

13. Popa I, and Dolan A I (2012). Numerical modeling of DC busbar contacts, 13th International Optimization of Electrical and Electronic Equipment, 188-193.

14. Saarimaki E, Paajanen $M$ et al. (2006). Novel heat durable electromechanical film: processing for electromechanical and electret applications, IEEE Transactions on Dielectrics and Electrical Insulation, vol 13(5), 963-972.
15. Sarnago H, Lucia O et al. (2012). Dual-mode-operation halfbridge resonant converter for improved-efficiency induction heating system, Twenty-Seventh Annual Applied Power Electronics Conference and Exposition, 2184-2188.

16. Yang F (2009). Current-induced thermal stresses in a metal cylinder, Journal of Applied Physics, vol 106(11), 113536 113536-6.

17. Nituca C (2013). Thermal analysis for a double sided linear induction motor, European Scientific Journal, vol 9, No. 9, $38-50$.

18. Liang C, Sun F P et al. (1992). Investigation of the energy transfer and consumption of adaptive structures, 31st IEEE Conference on Decision and Control, 1791-1796.

19. Aparaschivei A, Cantemir L et al. (2004). Consideration regarding electromagnetic differential with one stator two disk rotors and ring windings, Buletinul Institutului Politehnic Iaşi, Tomul 1 (LIV), 21-24.

20. Wei L, Young W et al. (2010). An Adaptive Equivalent Circuit Modeling Method for the Eddy Current-Driven Electromechanical System, IEEE Transactions on Magnetics, vol 46(6), 1859-1862.

21. Seung-Myen L, Se-Hee L et al. (2005). Reduced modeling of eddy current-driven electromechanical system using conductor segmentation and circuit parameters extracted by FEA, IEEE Transactions on Magnetics, vol 41(5), 1448-1451.

22. Vosskamper L M, Schmid R et al. (2000). Combining models of physical effects for describing complex electromechanical devices, IEEE/ACM International Workshop on Behavioral Modeling and Simulation, 42-45.

23. Cantemir L, Chiriac G et al. (1998). Some principles problems concerning the electromechanical drive, Proceedings of the $9^{\text {th }}$ National Conference on Electrical Drive, University of Craiova, 13-18.

24. Livinţ G, Horga V et al. (2010). Electrical drive systems control on a hybrid vehicle model, Buletinul Institutului Politehnic Iaşi, vol LVI(LX), 103-115. 